\title{
El tráiler cinematográfico y la subtitulación en la didáctica de lenguas: aplicación práctica en la combinación lingüística alemán - español
}

\author{
José Luis Martí Ferriol ${ }^{1}$ \\ Universitat Jaume I de Castelló
}

$\mathrm{M}^{\mathrm{a}}$ Rosario Martí Marco

Universitat d'Alacant

\begin{abstract}
This article describes a teaching experience carried out in the lectures of language for translators in the Degree of Translation and Interpreting at the Universidad de Alicante during the courses 2014-2015 and 2015-2016. The practice of both direct and reverse subtitling was used as a language learning strategy in the subject "Contrastes lingüísticos y culturales de la Lengua B (Alemán)". Practical exercises were done by using the subtitling program Subtitle Workshop 2.51, while the theoretical knowledge related to this audiovisual mode was extracted from a reference manual in German language (Jüngst, 2010), from which notes and a glossary in German had been elaborated for this specific purpose. The results of the evaluation by the students based on a survey, for both 2014-2015 and 20152016 editions, show their satisfaction. Students pointed out their interest for subtitling, as well as for the selected genre, which is proposed here as a most appropriate one for the desired didactic objectives. As mentioned above, this experience took place at the Universidad de Alicante, although the subtitling workshop was given by a lecturer from the Universitat Jaume I in Castellón.
\end{abstract}

Keywords: direct subtitling, reverse subtitling, movie trailer, audiovisual translation, advertising

Corresponding author - Universitat Jaume I, Departament de Traducció i Comunicació, Av. De Vicent Sos Baynat, s/n, 12071 Castelló de la Plana (Espanya).

Email: martij@trad.uji.es 


\section{RESUMEN}

Este artículo describe una experiencia docente llevada a cabo en la clase de lengua para traductores del Grado en Traducción e Interpretación de la Universidad de Alicante durante los cursos 2014-2015 y 2015-2016 2. En concreto, se ha utilizado la práctica de la subtitulación directa e inversa (alemán-español) de tráileres cinematográficos como mecanismo de aprendizaje de lenguas en la asignatura "Contrastes lingüísticos y culturales de la Lengua B (Alemán)". Las actividades prácticas se han llevado a cabo con el programa de subtitulación Subtitle Workshop 2.51, y los conocimientos teóricos impartidos sobre esta modalidad de traducción audiovisual se han extraído de un conocido texto sobre traducción audiovisual en lengua alemana (Jüngst, 2010), a partir del cual se habían preparado unas notas y un glosario en dicha lengua. Los resultados obtenidos mediante una encuesta de evaluación docente en las ediciones de 2014-2015 y 2015-2016 muestran la satisfacción del alumnado, que destacó su interés tanto por la práctica subtituladora como por el género empleado, que aquí se postula como un ejemplo de género publicitario idóneo para la finalidad perseguida. Como se ha indicado, esta experiencia se ha llevado a cabo en la Universidad de Alicante, pero el taller de subtitulación ha sido impartido por un profesor de la Universitat Jaume I de Castellón.

Palabras clave: subtitulación directa, subtitulación inversa, tráiler cinematográfico, traducción audiovisual, publicidad

\section{Introducción teórica}

La labor de contextualización necesaria para presentar la experiencia que se describe en este artículo implica la realización de un breve repaso de algunas disciplinas, que si bien se encuentran relacionadas entre sí, poseen solamente algunos puntos de intersección entre sus ámbitos. Debemos, por lo tanto, hacer mención a tres aspectos: a la utilización de la subtitulación en el aprendizaje de lenguas, al estudio de la traducción de la publicidad en su sentido más amplio (y de materiales audiovisuales) y su docencia, así como al tráiler cinematográfico como (sub)género específico dentro de la publicidad, y a su traducción (en este caso, su subtitulación). En consecuencia, se deben considerar estas tres actividades conjuntamente, y aplicarlas al par de lenguas alemán/español, para concretizar la actividad que se describe. Su especificidad ( $u$ originalidad) es consecuencia directa de la combinación de todos estos aspectos, de manera conjunta y simultánea. Se realiza una (necesariamente) sucinta revisión teórica de los tres aspectos mencionados a continuación.

2 Una primera versión reducida del taller, de 3 horas de duración, se impartió durante el curso 2013-2014. 
La utilización docente de la traducción audiovisual no es nueva. En concreto, el uso de materiales audiovisuales subtitulados en el aula como herramienta encaminada a mejorar las diferentes competencias en la adquisición de una lengua extranjera por parte de los alumnos es objeto de investigación y estudio desde hace más de una década. Son pioneros y muy citados, en este sentido, los trabajos de Danan (2004), y especialmente el de Neves (2004), que realizó experimentos de este tipo en cursos de traducción en Portugal. En ambos casos, se utilizó subtitulación interlingüística ${ }^{3}$, el mismo tipo de práctica que se ha empleado en la experiencia que se describe en este artículo.

Los resultados de una investigación similar en nuestro país fueron publicados hace algunos años por (Talaván Zanón, 2006). La autora pone de manifiesto los beneficios didácticos que se pueden derivar de este tipo de prácticas, y amplía el marco de creación de subtítulos para el caso de los intralingüísticos en lengua extranjera, así como de los interlingüísticos, tanto en traducción directa como inversa. De nuevo, son estos dos tipos de subtitulación interlingüística (en sentido directo e inverso) los que se han empleado en la experiencia que se describe en este artículo.

Una vez descubierto el potencial de la subtitulación en el aprendizaje de lenguas, se han llevado a cabo diferentes proyectos de investigación a nivel europeo que, con financiación comunitaria, han intentado obtener conclusiones generalizables y transferibles a la sociedad para este tipo de actividades, con la finalidad de ser puestas en práctica en el entorno multilingüe de la Unión Europea. Entre ellos, y sin ánimo de describirlos en detalle, destacan los tres proyectos siguientes, que se han desarrollado de forma secuencial: Learning via Subtitling: Software \& Processes for Developing Language Learning Material based on Film Subtitling, también conocido como "LeViS" (http://levis.cti.gr/), que finalizó en 2008; Subtitles and Language Learning, también conocido como "SLL", que finalizó en 2011, y que dio lugar a la publicación de Gambier, Caimi y Mariotti (2014); y el denominado "ClipFlair Project": Foreign Language Learning through Interactive Revoicing and Captioning of Clips (http://www.clipflair.net/), cuya plataforma sigue activa. En el caso de este último, su objetivo fundamental estaba más bien encaminado a la creación de materiales audiovisuales para el aprendizaje de lenguas, y no se centraba únicamente en la modalidad de subtitulación, sino que incluía materiales de casi todas las demás

Esta modalidad de traducción audiovisual supone el trasvase de contenidos entre dos lenguas diferentes. Es decir, que el contenido lingüístico de la versión original está formulado en una lengua, y los subtítulos generados a partir del mismo se presentan en modo escrito en una lengua diferente. En el caso de la subtitulación intralingüística (como la subtitulación para sordos y personas con deficiencias auditivas), tanto el contenido lingüístico como los subtítulos se representan en la misma lengua. 
modalidades de traducción audiovisual (doblaje, voces superpuestas, diferentes tipos de modalidades de accesibilidad, etc.).

A la hora de describir la actividad que nos concierne, debemos centrarnos en segundo lugar en la traducción de la publicidad. La revisión de toda la bibliografía dedicada a la traducción de la publicidad resultaría un ejercicio laborioso y muy complejo, que no pretendemos abordar dada la aproximación más bien práctica y didáctica de este artículo. Baste, entonces, con citar algunas referencias de libros dedicados a este asunto en nuestro entorno nacional. Para ello, hemos seleccionado los trabajos de Bueno García (2000), Valdés Rodríguez (2004) y Montes Fernández (2007), obras que son resultado de trabajos de investigación (en especial las dos últimas), y que incluyen gran cantidad de ejemplos prácticos muy visuales, donde se explican las diferentes estrategias de traducción que se suelen seguir a la hora de traducir publicidad, tanto en formato visual como audiovisual. El trabajo de Montes Fernández resulta especialmente aplicable en nuestro caso, ya que incluye la combinación lingüística que nos concierne.

Del mismo modo, la revisión de la bibliografía dedicada a la docencia de la traducción de la publicidad requeriría de una extensión superior a la de que se dispone. Además, dicha revisión se ha llevado a cabo recientemente, y de forma exhaustiva, por Chaume y Martí Ferriol (2014), publicación a la que nos remitimos para su repaso. La metodología docente propuesta en dicha obra sugiere una secuenciación en cinco pasos, dentro de la cual la subtitulación de publicidad audiovisual ocupa la tercera posición.

En tercer y último lugar de esta introducción revisamos a continuación la especificidad del tráiler como (sub)género dentro del lenguaje publicitario, al mismo tiempo que postulamos su idoneidad como material útil en la subtitulación como metodología de aprendizaje de lenguas.

Por lo que respecta al primero de los aspectos mencionados (su especificidad, en este caso narrativa), Jensen (2014, p. 105) ya menciona en el resumen de su artículo que:

The hypothesis is that trailers that follow a classic genre convention seek to sell their products by condensing a range of genre traits, which arouses a specific, intense emotional experience. Most particularly, the trailer chooses to activate the main genre of the film and the corresponding range of emotions by reducing and reordering its often complex narrative. On this basis, compared to the film, the trailer may be viewed as an alternative narrative. 
Del mismo modo, Hesford (2013, p. 1) aboga por las particularidades de expresión de este género:

[...] I will examine how a culture of digressive reading positions the film trailer as a new form of cinematic expression - one which subverts and exploits negative associations of commercialism as part of a performative effect and which carries implications for the wider theoretical discussion of cinema.

También en esta línea, se pronuncian López Font y Correia Baptista (2011), que para la definición y categorización del tráiler citan a dos autores españoles: Dornaleteche Ruiz (2007) y Martínez Puche (2008). Del primero de ellos, destacan su tipología, que los clasifica en seis tipos ${ }^{4}$. Para nuestro estudio, nos interesa especialmente el cuarto (ya que nuestro corpus de tres tráileres pertenecería a este tipo), que define como (2007, p. 108) "Theatrical trailer (tráiler "tradicional o (sic) standar" de cine). Es el modelo de tráiler por antonomasia proyectado en la sala de cine. Utiliza el montaje discontinuo de imágenes de la película que promociona". Del segundo de los autores, citan que, según él, este tipo de textos publicitarios tiene una doble naturaleza (2008, p. 80): "persuasiva; que incita, y narrativa; que resume y argumenta. El tráiler, pues, informa, crea expectación y transmite una estética y una temática". Martínez Puche también propone una tipología de clasificación, que tiene su criterio de clasificación principal basado en aspectos narrativos, y que tiene cuatro posibilidades 5 . Para nuestro caso, nos interesaría el "narrativo no lineal", caracterizado por la presentación de momentos inconexos. López Font y Correia Baptista (2011, p. 853) concluyen su repaso por la definición y caracterización del género indicando que "Resulta interesante la aplicación paralela del lenguaje publicitario, para aludir a la importancia de la influencia de la seducción y la interpretación en la construcción de sentido que va implícita al objetivo del tráiler".

Desde nuestro punto de vista, es esa combinación de la función apelativa característica del texto publicitario y de la necesidad de construir sentido a partir, normalmente, de fragmentos narrativos inconexos, lo que hace especialmente idóneo el tráiler como (sub)género para atraer la atención y el interés del estudiante. Nuestra experiencia constata que dicha combinación ejerce un efecto de

Los 6 tipos de tráileres son: Teaser Trailer (tráiler incitador), Creative trailer (tráiler creativo), Clip trailer (tráiler fragmento), Theatrical trailer (tráiler "tradicional o estándar" de cine), TV Trailer (tráiler en televisión que puede vincularse a marcas privadas) y Behind the scenes trailer (tráiler detrás de las cámaras).

5 Dichas posibilidades son las siguientes: narrativo lineal, narrativo no lineal, no narrativo o experiencial y autorreferencial. 
motivación muy significativo, lo que predispone favorablemente al estudiante a focalizar su esfuerzo en las tareas de subtitulación, a la asimilación de conceptos teóricos (en lengua extranjera) porque necesita entenderlos para poder crear subtítulos, así como al aprendizaje del uso del programa de subtitulación, que se hace necesario para poder llevar a cabo las actividades propuestas. La motivación surge desde el mismo momento en que los estudiantes visionan los tráileres que deben subtitular. Dicha predisposición se pone de manifiesto por medio de su participación activa en la tarea, y por la formulación de preguntas y comentarios constantes relacionados con los contenidos de los tráileres.

\section{Materiales y metodología}

La asignatura obligatoria "Contrastes lingüísticos y culturales de la Lengua B (Alemán)" se ofrece en el primer cuatrimestre del tercer curso del Grado de Traducción e Interpretación. La experiencia docente de cursos anteriores indica que los estudiantes españoles llegan en su gran mayoría a esta asignatura con un nivel de lengua alemana que suele situarse en B1-B2. En cuanto a los alumnos alemanes (Erasmus), en la edición de 2014-2015, eran estudiantes de Traducción en las Universidades de Hannover y Erlangen, con un nivel de lengua española entre A2 y C1. Además, había un alumno bilingüe y una estudiante alemana residente en España. En el caso del curso 2015-2016, participaron cuatro estudiantes Erasmus alemanas; una de ellas provenía de la Universität Leipzig, y las otras tres de Hildesheim.

La experiencia docente del taller de subtitulación interlingüística encaja perfectamente dentro del programa de la asignatura, entre cuyos objetivos generales se incluye la adquisición de conocimiento general y básico desde el punto de vista contrastivo de los grandes aspectos y fenómenos de la relación lingüística, literaria, traductológica y, en especial, cultural del mundo germánico e hispánico, así como el tratamiento teórico de los fenómenos comparatistas relevantes en la historia de la lengua y de la literatura alemanas y el tratamiento traductográfico de textos sobre cultura editorial actual y textos de interés puntual.

Entre los objetivos específicos de la asignatura, se pueden destacar los siguientes:

1. Adquirir un suficiente conocimiento general y básico, desde el punto de vista contrastivo, de los grandes aspectos y fenómenos de la relación literaria, lingüística y, en general, cultural del mundo germánico con el hispánico.

2. Reflexionar y comprender las realidades literarias de los países en los que se habla la Lengua B (alemán).

3. Valorar especialmente los textos literarios y el instrumental filológico. 
4. Profundizar en el conocimiento de la historia de la lengua, la lengua literaria y la cultura alemana.

5. Acceder a una perspectiva crítica.

6. Asimilar reflexivamente la realidad cultural de los países de habla alemana.

La experiencia docente del taller de subtitulación interlingüística se programó en un formato de dos sesiones con una duración total de cinco horas. Los contenidos, expresados de manera resumida, fueron los siguientes:

- Parte 1: Introducción general a las convenciones de subtitulación interlingüística comercial.

- Parte 2: Introducción al uso del programa de subtitulación Subtitle Workshop, programa de software de acceso libre en Internet.

- Parte 3a: Creación de subtítulos en español para el tráiler de la película alemana Gegen die Wand (Fatih Akin, 2004).

- Parte 3b: Creación de subtítulos en alemán para el tráiler de la película española Volver (Pedro Almodóvar, 2006).

- Parte 3c: Visualización de tráiler en alemán subtitulado en español de Oh Boy (Jan Ole Gerster, 2012).

Las partes 1 y 2 se impartieron en una primera sesión de dos horas. La Parte 3 ( $a$, b y c) se llevó a cabo al día siguiente en una segunda sesión de tres horas (una hora para la Parte 3a, una hora para la Parte 3b, y una última hora para la Parte 3c).

Para la documentación de la Parte 1, que se puede considerar como una introducción teórica a las convenciones de subtitulación, se tomó como referencia el libro de Jüngst (2010), dedicado a la traducción audiovisual. En concreto, los materiales entregados a los estudiantes fueron unas notas y un glosario que se obtuvieron del capítulo de la obra que la autora alemana dedica a la subtitulación interlingüística (Interlinguale Untertitelung, capítulo 2, páginas 25-58). Estos contenidos se materializaron en forma de un fichero de texto de 4 páginas, que contenía los principales aspectos teóricos y terminológicos relacionados con la actividad de la subtitulación interlingüística. De esta forma, los alumnos se vieron expuestos a estos conceptos, que en su mayoría veían por primera vez, en lengua alemana (como, por ejemplo, términos específicos de la subtitulación, como 6Sekunde-Regel o Lesegeschwindigkeit; o términos de lenguaje común, como Zeilen o Zeichen, aplicados a este ámbito temático). Además de aprender la terminología, los alumnos se vieron obligados a asimilar los conceptos tal y como se mostraban escritos en la lengua origen, aunque la mayor parte de las explicaciones adicionales por parte del docente se realizaron en lengua meta. Se insistió especialmente en la importancia de dos conceptos que son claves en la creación de subtítulos: 
Auslassung (omisión o eliminación de contenidos), y Verdichtung (síntesis de la información).

En esta parte también se llevaron a cabo ejercicios de traducción a la vista, ya que se animó a los estudiantes a que leyeran y reformularan, en voz alta y en español, algunos de los contenidos de la documentación entregada, que, como se ha indicado, tenían que ver con las convenciones propias de esta modalidad de subtitulación y estaban redactados en lengua alemana.

El curso se impartió en un aula de informática, donde cada alumno disponía de su ordenador. Los estudiantes tenían acceso a un directorio compartido en un servidor donde, entre los materiales del curso, disponían de una versión comprimida del programa de subtitulación Subtitle Workshop 2.51, aunque dicho programa es de acceso libre en Internet (https://subtitle-workshop.uptodown.com/windows).

La Parte 2 del curso se inició con la instalación del programa de subtitulación, y el docente explicó las acciones más sencillas pero imprescindibles para poder iniciar los trabajos de localización y creación de los subtítulos, así como las necesarias para poder almacenar los subtítulos una vez realizados. La utilización de este programa de subtitulación es muy sencilla e intuitiva, y los alumnos son capaces de comenzar a crear sus propios subtítulos después de una explicación de no más de 5 o 10 minutos. Aunque existen manuales de usuario para este programa en Internet, no se consideró necesario incluir ninguno de ellos entre los materiales del curso, dada la limitación temporal de la docencia y la sencillez de uso mencionada. Al finalizar la sesión se animó a los alumnos a practicar con el programa en casa, pues en la siguiente sesión (al día siguiente), deberían generar subtítulos para los dos tráileres, en las partes $3 a$ y $3 b$.

Como materiales para las partes $3 a$ y $3 b$ los alumnos disponían del programa de subtitulación, donde cargaron el vídeo de cada uno de los dos tráileres (el de Gegen die Wand y el de Volver), además de un fichero con las listas de diálogos de cada uno de los dos textos audiovisuales. Primero se realizó durante una hora la traducción directa del tráiler de la película de Fatih Akin ${ }^{6}$, y a continuación se llevó a cabo durante otra hora la generación de subtítulos en alemán para el tráiler de la película de Pedro Almodóvar.

La metodología seguida fue, en primer lugar, dejar a los alumnos que crearan sus subtítulos individualmente. Después de unos 40 minutos, aproximadamente, se procedió a revisar de forma conjunta las propuestas de subtítulos de algunos

6 Aunque había en la clase algunos estudiantes Erasmus cuya lengua materna era el alemán, la mayoría de los alumnos eran españoles. 
alumnos (dos o tres, dependiendo de la disponibilidad de tiempo). Durante dicha revisión se enfatizó en la importancia de la práctica de la síntesis, característica intrínseca y fundamental de la subtitulación, y especialmente significativa en esta combinación lingüística. Los alumnos tuvieron algunos problemas al principio con este aspecto, pero se tuvieron que esforzar para reformular sus traducciones de la forma más sintética posible. Este es un ejercicio especialmente apropiado para la adquisición de competencias específicas en el aprendizaje de una lengua extranjera (traducción inversa), y en el análisis contrastivo de las dos lenguas (traducción directa e inversa), ya que en la práctica de la síntesis los alumnos deben seleccionar la información más importante y buscar reformulaciones que ocupen menos caracteres en los subtítulos.

A continuación se muestra (en formato texto) algunos ejemplos de la propuesta de subtítulos presentada por el profesor para la traducción del tráiler de la película de Fatih Akin ${ }^{7}$ al finalizar la Parte 3a. El vídeo del tráiler de Gegen die Wand comienza con un breve diálogo entre dos personajes que incluye las siguientes intervenciones:

- Es gibt tausend andere Möglichkeiten sich das Leben zu nehmen. Warum fahren Sie gegen eine Wand?

- Wer sagt Ihnen, dass Ich mich umbringen wollte?

- Es gab keine Bremspuren.

Como se puede apreciar, se trata de un diálogo que puede tener cierta complejidad y que, además, se pronuncia a una velocidad elevada. Para el pautado de los subtítulos, se ha utilizado un subtítulo por cada una de las oraciones, utilizando las pausas naturales que existen entre ellas (esto es fácil de asimilar por los alumnos, que así comienzan su labor de pautado y creación de subtítulos en esta actividad). Sin embargo, como puede comprobarse en los subtítulos mostrados a continuación, ha sido necesario realizar una labor de síntesis importante, especialmente en el último subtítulo de la serie, que apenas alcanza una duración de un segundo. Aquí los estudiantes deben poner a prueba su capacidad para reformular una traducción literal, que sería "No había marcas de frenado" (que no se podría leer), y utilizar en este caso una modulación (o cambio del punto de vista), para proponer una solución con solo dos palabras ("No frenó"), que sí daría tiempo a leer.

$7 \quad$ La lista de diálogos del tráiler y la propuesta completa de subtítulos se muestran en el Anexo. 
00:00:02,501 --> 00:00:04,933

Hay muchas formas

de quitarse la vida.

2

00:00:05,367 --> 00:00:06,900

¿Por qué contra la pared?

3

00:00:07,801 --> 00:00:09,801

¿Quién dice que me quería matar?

4

00:00:10,801 --> 00:00:11,867

No frenó.

A continuación se muestra (en formato texto) una propuesta presentada por el profesor de dos subtítulos en la traducción del tráiler de la película de Pedro Almodóvar ${ }^{8}$ al finalizar la Parte 3b. En este caso, se muestran dos ejemplos donde es necesaria no solo la labor de síntesis, sino también la de omisión, ya que todo el diálogo original no puede reproducirse en el subtítulo. Se trata de las dos intervenciones siguientes, que se formulan de forma muy rápida en unos cinco segundos ${ }^{9}$, en dos momentos diferentes del tráiler:

- Estaba contando lo de mi Borja, que me lo han vuelto a apercibir de expulsión. ¿Qué te parece? Le ha pinchado una rueda al cura que le da religión.

- Estaba ciega con tu padre. Y él se aprovechó, y me puso los cuernos hasta el último día de su vida.

Como se puede comprobar, se trata de dos fragmentos con mucha información, que incluyen varias oraciones principales y subordinadas. Ante la imposibilidad de subtitular todo el contenido, el alumno deberá entender que debe ser capaz de seleccionar la más importante. En el primer caso, que Borja ha sido expulsado o que ha recibido un aviso (Verweis), y que ha pinchado una rueda (einen Reifen zerstochen), sin decir a quién ("el cura de religión") y sin indagar la opinión del receptor ("¿Qué te

$8 \quad$ La lista de diálogos del tráiler y la propuesta completa de subtítulos se muestran en el Anexo.

9 En el taller impartido se utilizó un pautado máximo de 5 segundos, para un subtítulo de dos líneas de cómo máximo 35 caracteres cada una. Estos son los criterios que se siguen para la docencia de la subtitulación interlingüística en la Universitat Jaume I. 
parece?"'). En el segundo, los fragmentos más importantes serían que había estado ciega (blind), y que le había puesto los cuernos hasta el último día (er ist fremdgegangen bis zum letzten Tag), mientras que se podría dejar sin subtitular que él "se aprovechó". Las propuestas de subtítulos en alemán para estos fragmentos son las siguientes, ambas de casi cinco segundos de duración, y ambas de dos líneas de unos 35 caracteres cada una.

12

00:00:34,534 --> 00:00:39,267

Borja hat ein Verweis bekommen.

Er hat einen Reifen zerstochen.

19

00:00:55,334 --> 00:00:59,267

Ich war blind, er ist fremdgegangen

bis zum letzten Tag!

Para finalizar, en la tercera hora de la sesión (Parte 3c), se mostró a los alumnos el tráiler en VO subtitulada en español de una película alemana reciente (2012): Oh $B o y^{10}$. La finalidad de este ejercicio era doble:

- por un lado, se hizo comprobar a los alumnos que las características de un producto comercial final subtitulado, del mismo género que ellos habían trabajado, eran muy similares a las de los subtítulos que ellos habían producido (aplicación profesional de la docencia).

- por otro lado, se compararon los diálogos en alemán con los subtítulos en español del tráiler. Como entre los materiales para esta parte no se disponía de la lista de diálogos del texto audiovisual original, se puso a prueba la capacidad de comprensión oral de los alumnos. Además, se llevaron a cabo ejercicios de traducción directa, donde se comentaron las soluciones empleadas por el subtitulador del tráiler. Se incidió (pero esta vez los alumnos pudieron comprobar por medio de un producto comercial que la realidad es esa) en la imposibilidad de transcribir literalmente en lengua meta los diálogos en lengua original y se constató, de nuevo, que la síntesis (Verdichtung) e, incluso, la omisión de información (Auslassung) son estrategias imprescindibles en la práctica de la subtitulación, especialmente en el caso de esta combinación lingüística (aplicación docente específica para la práctica de la traducción).

10 El tráiler subtitulado de esta película puede visualizarse en la dirección que aparece en el apartado de Filmografía, al final de este artículo. 
La utilización del tráiler en esta parte del taller se completó a continuación con la presentación de un texto en alemán como documentación adicional. Se trata de un texto que realiza una crítica de la película en cuestión y que puede encontrarse en la web www.ohboy.x-verleih.de/.

Además, en la siguiente clase de la asignatura "Contrastes lingüísticos y culturales de la Lengua B (Alemán)", la profesora a su cargo trabajó con los estudiantesespectadores-traductores dicho texto de la tragicomedia Oh Boy (2012) en alemán, enmarcándola en su trama original y contexto berlinés, dado que la película despliega varios recursos que incrementan su estética, como la iconografía en blanco y negro y la música de jazz. En este sentido, se hizo un balance de los contenidos teóricos relacionados con el taller que los alumnos debían estudiar para el examen final.

En cualquier caso, resultó evidente que la continua experimentación en la "pantalla grande" despierta gran interés entre el alumnado y que las técnicas de subtitulación les descubren un nuevo panorama profesional con destrezas que pueden implementar.

\section{Resultados}

Con la intención de obtener información empírica de esta propuesta de metodología docente, se distribuyó un cuestionario entre los alumnos que asistieron al taller de subtitulación: un total de 14 estudiantes en el curso 2014-2015, y de 14 estudiantes en la edición del curso 2015-2016.

El cuestionario repartido correspondía a un formato estándar que se emplea en la Universitat Jaume I para cursos impartidos por personal externo, y que consta de 12 preguntas, que se deben evaluar en una escala (de Lickert) de 1 a 5 puntos, donde el valor 1 corresponde a "muy bajo", y el valor 5 corresponde a "muy alto". Además, el cuestionario incluye una casilla en blanco donde el estudiante debe reflejar, también con un valor numérico del 1 al 5, su satisfacción general con el curso.

Las doce preguntas interrogan sobre los siguientes aspectos: (1) relevancia del curso para el trabajo, (2) calidad del contenido, (3) organización y estructura del contenido, (4) claridad expositiva del monitor, (5) capacidad de motivación del monitor, (6) dominio del contenido del monitor, (7) adecuación de los materiales empleados, (8) estímulo a la participación y a la reflexión, (9) relación del monitor con los asistentes, (10) adquisición de nuevas destrezas, (11) posibilidad de aplicación práctica y (12) adecuación a los objetivos del programa. 
Los resultados cuantitativos de satisfacción general (que se muestran en la tabla 1, a continuación) arrojaron un valor de la media de 4,14 puntos, con una desviación estándar de 0,66 puntos en el caso de la edición de 2014-2015; y un valor de la media de 4,93 puntos, con una desviación estándar de 0,27 puntos en el caso del curso 2015-2016. Esto quiere decir que la satisfacción general fue más que alta (a la que correspondería un valor de 4) en el primer caso, y prácticamente muy alta en el segundo caso.

La tabla anónima con los resultados numéricos obtenidos de la encuesta se presenta a continuación:

\begin{tabular}{lll}
\hline Alumno & $\begin{array}{l}\text { Puntuación } \\
(2014-2015)\end{array}$ & $\begin{array}{l}\text { Puntuación } \\
(\mathbf{2 0 1 5 - 2 0 1 6 )}\end{array}$ \\
\hline 1 & 4 & 4 \\
\hline 2 & 3 & 5 \\
\hline 3 & 4 & 5 \\
\hline 4 & 4 & 5 \\
\hline 5 & 4 & 5 \\
\hline 6 & 5 & 5 \\
\hline 7 & 5 & 5 \\
\hline 8 & 5 & 5 \\
\hline 9 & 4 & 5 \\
\hline 10 & 4 & 5 \\
\hline 11 & 3 & 5 \\
\hline 12 & 4 & 5 \\
\hline 13 & 5 & 5 \\
\hline 14 & 4 & 5 \\
\hline
\end{tabular}

Tabla 1. Resultados de los cursos 2014-2015 y 2015-2016.

Además, el cuestionario se completa con una serie de dos preguntas abiertas, que los estudiantes pueden rellenar con sus comentarios personales. La primera de ellas interroga sobre los "Aspectos positivos y negativos", mientras que la segunda pregunta sobre "Aspectos nuevos a añadir o a profundizar más, sugerencias y modificaciones". Para la primera de las dos preguntas, algunos comentarios de los estudiantes del curso 2014-2015 fueron las siguientes:

- Me parece un curso muy útil, pero un poco corto para mi futuro, porque es un tema que me interesa para mi futuro.

- El material y los conocimientos adquiridos pueden ser muy positivos y útiles en un futuro laboral.

- Lo que más me ha gustado ha sido la práctica y la posibilidad de practicar con un programa que se usa para la subtitulación en la vida real. 
- Nos ha servido para conocer más ramas del mundo del traductor y como una cosa que parece tan sencilla, en realidad es bastante compleja y creativa.

- M'ha encantat el curs, m'agrada molt la traducció audiovisual i sobretot les pel-lícules. Ha sigut una experiència molt bona.

Comentarios similares, en este caso realizados por los alumnos del curso 2015-2016, son los siguientes:

- Ha sigut molt útil i interessant.

- Como persona que quiere dedicarse a la traducción audiovisual, el curso me ha parecido muy útil, como introducción al tema. La única pega que le pondría, es que me hubiera gustado que fueran más horas. Ha sido muy interesante.

- Más asignaturas como esta deberían ser obligatorias para el grado dado que tiene tanto comprensión oral como escrita de la lengua.

- Ha sido un curso muy entretenido y sobre todo interesante ya que nunca había probado a subtitular y no sabía como (sic) era y si me gustaba. Ahora la tendré como opción en el futuro.

- -Las películas seleccionadas. -Tanto subtitulación directa como inversa. Análisis de las traducciones de los participantes.

Para la segunda de las preguntas, algunos comentarios de los estudiantes de la edición de 2014-2015 fueron:

- Estructuración más adecuada del tiempo y nivel de los alumnos.

- Hemos profundizado lo máximo posible, ya que el tiempo ha sido limitado.

- Hacer más hincapié en la traducción directa que en la inversa, ya que hay más posibilidades de dedicarnos a ello que a la inversa.

- Pense que seria bona idea repetir el curs per l'any que ve.

- Introducir doblaje (aunque en las horas que dimos no daba tiempo).

Comentarios similares, en este caso realizados por los alumnos del curso 2015-2016, son los siguientes:

- Treballar més en aspectes com la omissió (sic) o condensar els textos.

- Profundizar más en la omisión y síntesis de los subtítulos, ya que es lo más difícil.

- El taller es demasiado interesante como para que dure solamente 5 horas, sería genial tener la oportunidad de hacer algo más extenso.

- Por mi parte, todo ha sido perfecto, pero se me ha hecho muy corto, si tuviera más horas, mucho mejor. 
- El programa lo veo correcto y bien estructurado. El problema ha sido la brevedad.

Como puede verse, la satisfacción general de los alumnos fue alta, también, por lo que se desprende de los resultados de la evaluación cualitativa. El aumento de la satisfacción (en términos cuantitativos) en la segunda edición podría explicarse por el hecho de la repetición del taller, así como por la redacción de este artículo (realizada en su mayoría entre las dos ediciones, pero completada después de la segunda), por lo que pudo suponer de ejercicio de reflexión e interiorización sobre esta actividad docente.

\section{Conclusiones}

A la vista de la información obtenida de los estudiantes, y presentada por medio de resultados cuantitativos y cualitativos, pensamos que se puede obtener conclusiones positivas de esta experiencia, pues los estudiantes se mostraron satisfechos con los contenidos y con su utilidad.

Se comprueba, del mismo modo que en estudios empíricos anteriores (Neves, 2004; Talaván Zanón, 2006), que la subtitulación (o la creación de subtítulos) es una herramienta de gran utilidad en las clases de aprendizaje de lenguas, también en los cursos de formación para traductores. Esta modalidad de traducción audiovisual permite a los alumnos constatar que sus estudios tienen una aplicación práctica profesional, con la que pueden generar un producto acabado que puede ser fundamental en las labores de entretenimiento y de transmisión de conocimiento.

En cuanto a la utilización del (sub)género tráiler de película como material audiovisual a subtitular, pensamos que sus características lo hacen especialmente recomendable para una práctica docente de este tipo: tanto por su estructura narrativa, que llama la atención y atrapa inmediatamente al espectador (en este caso al estudiante), como por su carácter primordial de texto publicitario en sí mismo, donde la utilización de la lengua pone de manifiesto dificultades en el proceso de traducción, que pueden ayudar a mejorar su adquisición.

Desde nuestro punto de vista, ambos componentes potencian la motivación en el proceso de aprendizaje, y esta es sin duda una de las variables más importantes a tener en cuenta en la didáctica de lenguas extranjeras. 


\section{About the authors}

José Luis Martí Ferriol se licenció en Ciencias Químicas (Química Industrial) en la Universidad de Valencia. Durante años compaginó su trabajo en empresas de producción de proceso químico con su formación en Traducción e Interpretación en la Universitat Jaume I de Castelló, donde obtuvo tanto la licenciatura como el doctorado con premio extraordinario. Desde 2007 ejerce como investigador y docente a tiempo completo en esa misma universidad. Su investigación se ha centrado fundamentalmente en la traducción audiovisual. Entre sus principales publicaciones destacan los libros Cine independiente y traducción (2010, Valencia: Tirant Lo Blanch) y El método de traducción: doblaje y subtitulación frente a frente (2013, Publicacions de la Universitat Jaume I), así como varias contribuciones en revistas nacionales e internacionales.

María Rosario Martí Marco es Profesora Titular del Área de Filología Alemana en la Universidad de Alicante. Es especialista en humanismo germánico, literatura de paisaje y en la obra de Wilhelm y Alexander von Humboldt, sobre quienes ha publicado numerosos estudios (Wilhelm von Humboldt y la creación del sistema universitario moderno, 2012) y de quienes ha traducido y editado en español textos como el Ensayo político sobre la isla de Cuba (2004), el Diario de A. v. Humboldt en España (2011) y el Ensayo político sobre Nueva España (2016). Ha publicado en 2015 la obra: Karl Vossler. Positivismo e Idealismo en la ciencia del lenguaje. Una investigación lingüístico-filosófica. Ha sido miembro de diversos proyectos de investigación en los ámbitos de la Germanística, Teoría de la Literatura y Comparatismo.

\section{Article history}

Paper received: $1^{\text {st }}$ May 2015

Paper received in revised form and accepted for publication: $11^{\text {th }}$ December 2015

\section{Referencias}

Bueno García, A. (2000). Publicidad y Traducción. Soria: Vertere, Monográficos de la revista Hermeneus, $\mathrm{n}^{\circ} 2$.

Chaume, F. \& Martí Ferriol, J.L. (2014). Teaching advertising translation: A didactic proposal. Linguae - Revista de la Sociedad Española de Lenguas Modernas, 1, 75-98. 
Danan, M. (2004). Captioning and subtitling: Undervalued language learning strategies. Meta, 49(1), 67-77.

Dornaleteche Ruiz, J. (2007). Definición y naturaleza del trailer cinematográfico. Pensar la publicidad, 1(2), 99-116.

Gambier, Y., Caimi, A. \& Mariotti, C. (Eds.). (2014). Subtitles and Language Learning Principles, strategies and practical experiences. Bern, Berlin, Bruxelles, Frankfurt am Main, New York, Oxford, Wien: Peter Lang.

Hesford, D. (2013). 'Action!... Suspense!... Emotion!' The trailer as cinematic performance. Frames Cinema Journal <http://framescinemajournal.com/article/action-suspenseemotion-the-trailer-as-cinematic-performance/>.

Jensen, C. S. (2014). Reduced Narration, Intensified Emotion: The Film Trailer. Projections, $8(1), 105-125$.

Jüngst, H. (2010). Audiovisuelles Übersetzen: Ein Lehr- und Arbeitsbuch. Tübingen: GünterNarr.

López Font, L. \& Correia Baptista, C. M. (2011). Hibridación y generación de contenido en los tráileres y la comunicación on-line de Toy Story 3: Un ejemplo de objetivos multidimensionales. Actas IV Congreso Internacional sobre Análisis Fílmico. Nuevas tendencias e hibridación de los discursos audiovisuales en la cultura digital moderna. Universitat Jaume I de Castellón, Castellón 4-6 mayo de 2011. Madrid: Ediciones de Ciencias Sociales. <http://repositori.uji.es/xmlui/handle/10234/31256>.

Martínez Puche, S. (2008). El tráiler de cine: entre el spot publicitario y la demostración narrativa. Revista Nau de Comunicação Audiovisual, 1(2), 59-81.

Montes Férnandez, A. (2007). Traducción y globalización. Análisis y perspectivas del fenómeno publicitario (Inglés-Español-Alemán). Granada: Comares.

Neves, J. (2004). Language awareness through training in subtitling. In P. Orero (Ed.), Topics in Audiovisual Translation (pp. 127-140). Amsterdam, Philadelphia: John Benjamins.

Talaván Zanón, N. (2006). Using Subtitles to Enhance Foreign Language Learning. Porta Linguarum: revista internacional de didáctica de las lenguas extranjeras, 6, 41-52.

Valdés Rodríguez, C. (2004). La traducción publicitaria: Comunicación y Cultura. Valencia: Universitat de Valéncia, Servei de Publicacions. 


\section{Filmografía}

Tráileres comerciales de:

Gegen die Wand (Fatih Akin, 2004): VO en alemán.

https://www.youtube.com/watch?v=gGd6RaoYPxo

Volver (Pedro Almodóvar, 2006): VO en español.

https://www.youtube.com/watch?v=hp4u67AV8VI

Oh Boy ( Jan Ole Gerster, 2012): VO en alemán con subtítulos en español.

https://www.youtube.com/watch?v=fhyvwb3L7fo

\section{Anexo}

\section{Lista de diálogos del tráiler de Gegen die Wand}

-Es gibt tausend andere Möglichkeiten sich das Leben zu nehmen. Warum fahren Sie gegen eine Wand?

-Wer sagt Ihnen, dass Ich mich umbringen wollte?

- Es gab keine Bremspuren.

wenn du am ende bist

wenn du keine hoffnung mehr hast

wenn du am wenigsten erwartest

dann trifft sie dich

(Las cuatro líneas anteriores corresponden a textos que aparecen en pantalla, con la tipografía indicada)

Ich schlag dir in die Fresse, du Wichser.

-Könntest du mich heiraten?

-Fick dich.

-Wir tun doch nur so, als wären wir verheiratet, verstehst du?

-Wieso gerade ich, ich bin doch Penner.

-Weil meine Eltern dich akzeptieren würden, du bist türke Mann.

-Du falsche Fotze. 
-Das bin Ich.

-Du liebst sie?

-Ja, ich lieb' sie.

Wenn wir es tun, dann bin Ich deine Frau und du bist mein Mann.

GEGEN DIE WAND

Fatih Akin

(Texto en pantalla)

Wenn Sie Ihr Leben beenden wollen, dann beenden Sie doch Ihr Leben. Aber dafür müssen Sie doch nicht sterben.

\section{Propuesta de subtítulos en español para Gegen die Wand}

1

00:00:02,501 --> 00:00:04,933

Hay muchas formas

de quitarse la vida.

2

00:00:05,367 --> 00:00:06,900

¿Por qué contra la pared?

3

00:00:07,801 --> 00:00:09,801

¿Quién dice que me quería matar?

4

00:00:10,801 --> 00:00:11,867

No frenó.

5

00:00:13,667 --> 00:00:15,400

CUANDO ESTÁS EN LAS ÚLTIMAS

6

00:00:16,567 --> 00:00:18,100

CUANDO NO HAY ESPERANZA 
7

00:00:19,434 --> 00:00:20,901

CUANDO MENOS LO ESPERAS

8

00:00:21,967 --> 00:00:23,001

APARECE ELLA

9

00:00:26,801 --> 00:00:28,400

Te voy a partir la cara.

10

00:00:35,167 --> 00:00:36,534

¿Te casarías conmigo?

11

00:00:38,434 --> 00:00:39,500

¡Que te den!

12

00:00:40,734 --> 00:00:43,133

Fingiremos estar casados,

¿entiendes?

13

00:00:43,400 --> 00:00:45,701

¿Por qué yo?

Soy un muerto de hambre.

14

00:00:45,901 --> 00:00:48,167

Mis padres te aceptarían.

Eres turco.

15

00:00:55,801 --> 00:00:57,501

-Eres un puta.

-Esa soy yo. 
16

00:01:06,033 --> 00:01:11,042

-¿La quieres?

-Sí... la quiero.

17

00:01:13,801 --> 00:01:16,534

Si lo hacemos, seré tu mujer

y tú mi marido.

18

00:01:31,167 --> 00:01:32,819

CONTRA LA PARED

19

00:01:35,818 --> 00:01:37,971

Si quiere acabar con su vida, adelante.

20

00:01:38,206 --> 00:01:39,801

Pero no hace falta morir.

\section{Lista de diálogos del tráiler de Volver}

-¿Hay más cosas que yo debería saber, y que no sé?

-Mogollón.

-Hay gente que dice que la ha visto. ¿A ti no se te ha aparecido?

-No. ¿Y a ti?

-Tampoco.

-Mamá, hay algo que quieres que yo haga, ¿no?

- Cortarme el pelo.

Somos una familia pobre, pues viviremos como una familia pobre. Paula, cuelga ese teléfono ahora mismo.

Agustina tiene cáncer. Tienes cáncer, Agustina. Venga, un fuerte aplauso para Agustina. 
Estaba contando lo de mi Borja, que me lo han vuelto a apercibir de expulsión. ¿Qué te parece? Le ha pinchado la rueda al cura que le da religión.

Uy, qué olor a pedo. Si es como si acabara de estar mamá, peyéndose a culo lleno. ¿No lo oléis vosotras?

¿¿Queréis un poquito?

-No, no, no, no.

-Que la maría es mía. Mirad lo hermosas que están las plantas.

-No, no, no.

-Estaba ciega con tu padre. Y él se aprovechó, y me puso los cuernos hasta el último día de su vida.

-¿Se nos va a aparecer?

-Espero que no.

-Bueno, y si no pasará un pan con pan. Entonces, ¿qué es lo que me estás pidiendo? -Acompañarme hasta el río, abrir una zanja, enterrar el frigorífico y no hacer preguntas.

¡Ay, Dios mío!

-¿Y la rusa?

-¿Quién es la rusa?

-Una, que tengo para ayudarme.

No te muevas ni hables, que está mi madre.

Volver...

(Canción)

Es muy doloroso, que una hija no quiera a su madre.

...con la frente marchita. Las nieves del tiempo platearon mi sien.

(Canción)

\section{Propuesta de subtítulos en alemán para Volver}

1

00:00:01,434 --> 00:00:05,367 
Noch was, das ich wissen sollte?

-Vieles.

2

00:00:05,567 --> 00:00:08,267

EL DESEO PRÄSENTIERT

3

00:00:09,300 --> 00:00:10,834

Sie ist gesehen worden.

4

00:00:11,267 --> 00:00:12,767

Ist die dir erschienen?

5

00:00:13,001 --> 00:00:15,234

Nein. Und dir?

-Auch nicht.

6

00:00:16,200 --> 00:00:21,167

Mutter, soll ich etwas machen?

-Schneide mir die Haare.

7

00:00:21,801 --> 00:00:24,100

Wir sind arm,

und so leben wir.

8

00:00:24,401 --> 00:00:26,033

Den Hörer sofort auflegen!

9

00:00:26,634 --> 00:00:28,100

Agustina hat Krebs.

10

00:00:29,834 --> 00:00:30,968

Du hast Krebs. 
11

00:00:31,334 --> 00:00:33,167

Ein großer Applaus für sie!

12

00:00:34,534 --> 00:00:39,267

Borja hat ein Verweis bekommen.

Er hat einen Reifen zerstochen.

13

00:00:41,934 --> 00:00:43,001

Dieser Gestank...

14

00:00:44,033 --> 00:00:47,400

Als ob Mamá gerade richtig

einen hätte fahren lassen!

15

00:00:48,968 --> 00:00:49,968

Möchtet ihr?

16

00:00:50,267 --> 00:00:51,367

Nein.

-Nein.

17

00:00:51,701 --> 00:00:53,834

Mein Gras.

Die Pflanze sind schön.

18

00:00:54,100 --> 00:00:55,133

Nein.

19

00:00:55,334 --> 00:00:59,267

Ich war blind, er ist fremdgegangen

bis zum letzten Tag! 
20

00:00:59,667 --> 00:01:01,501

Wird sie erscheinen?

-Nein.

21

00:01:02,234 --> 00:01:04,801

Worum geht es?

22

00:01:05,100 --> 00:01:07,567

Um mich zu begleiten,

eine Grube zu tun,

23

00:01:08,200 --> 00:01:09,901 zu vergraben, ohne Fragen.

24

00:01:10,133 --> 00:01:11,167

Mein Gott!

25

00:01:11,501 --> 00:01:13,701

Und die Russin?

-Die Russin?

26

00:01:14,968 --> 00:01:17,067

Eine, die mir hilft.

27

00:01:17,300 --> 00:01:19,367

Keine Bewegung!

Die Mutter ist da.

28

00:01:26,100 --> 00:01:27,934

$<$ i $>$ Zurückkehren...</i> 
29

00:01:28,167 --> 00:01:31,500

Es tut weh, wenn eine Tochter ihre Mutter nicht liebt.

30

00:01:33,334 --> 00:01:35,234

$<$ i $>$ mit runzliger Stirn. $</$ i $>$

31

00:01:35,534 --> 00:01:39,267

$<i>$ Der Schnee der Zeit

versilberte meine Schläfe. $</ \mathrm{i}>$ 